\title{
L'impact d'un couvert de glace sur la dynamique des niveaux et débits dans un estuaire méso tidal
}

\author{
Brian Morse ${ }^{\mathrm{a}}$ et Benoîte Ringô ${ }^{\mathrm{b}}$ \\ ${ }^{a}$ Université Laval, Québec, Canada,Brian.Morse@gci.ulaval.ca \\ ${ }^{b}$ Antérieurement Université Laval, maintenant Safège Cetiis, Aix en Provence, France, \\ benoite_ringo@hotmail.com
}

\section{Résumé}

Cet article présente une modélisation numérique (1-D) à éléments finis de l'estuaire de la rivière Portneuf, Québec (profondeur moyenne de $3 \mathrm{~m}$ et $5,9 \mathrm{~km}$ de long). Les marnages de $1,8 \mathrm{~m}$ (marées mortes) et de $3.0 \mathrm{~m}$ (marées vives) sont amortis en présence d'un couvert de glace. Proche du barrage (5,6 km de l'embouchure) l'atténuation varie entre $17 \%$ et $37 \%$ respectivement. Les débits engendrés lors du flot (flux) par les marées mortes et vives sont typiquement $200 \mathrm{~m}^{3} / \mathrm{s}$ et $500 \mathrm{~m}^{3} / \mathrm{s}$ à l'embouchure. Lorsqu'il y a couvert de glace, ces valeurs sont atténuées de $30 \mathrm{~m}^{3} / \mathrm{s}$ à $100 \mathrm{~m}^{3} / \mathrm{s}$ respectivement, alors qu'au jusant, l'atténuation est d'environ $50 \mathrm{~m}^{3} / \mathrm{s}$ indépendamment du marnage.

\begin{abstract}
$\underline{\text { Abstract }}$
This article is the fruit of three years of observations on the Portneuf Estuary, Québec (5.9 $\mathrm{km}$ in length and $3 \mathrm{~m}$ deep in the main channel). According to a one-dimensional finite element numerical model, the presence of an ice cover on the estuary causes an attenuation of the neap tidal range $(1.8 \mathrm{~m})$ and spring tidal range $(3.0 \mathrm{~m})$ of $17 \%$ and $37 \%$ respectively near the upstream end of the estuary (at the dam). The 200 (flood neap tide) and $500 \mathrm{~m}^{3} / \mathrm{s}$ (flood spring tide) flowrate at the mouth is also attenuated by the ice cover by 30 and $100 \mathrm{~m}^{3} / \mathrm{s}$ respectively. During the ebb tide, the attenuation is about $50 \mathrm{~m}^{3} / \mathrm{s}$ during both neap and spring tides.
\end{abstract}

\section{Introduction}

Plusieurs estuaires nordiques sont parfois recouverts d'un couvert de glace pendant une certaine période durant l'hiver. Très peu est connu de l'impact que ce couvert pourrait avoir sur les niveaux, les débits et les courants (Morse et al. 1999). Basée sur trois hivers de relevés sur un estuaire québécois, et une simulation unie dimensionnelle non permanente, cet article quantifie l'impact du couvert sur l'écoulement et présente des explications physiques.

Les études se sont réalisées grâce au soutien financier et technique pendant trois ans de Hydro Québec (Morse et al. 2001, 2002, 2003).

\section{$\underline{\text { 2.Notions théoriques }}$}

\subsection{Influence d'un couvert de glace sur l'écoulement dans une rivière}

L'impact d'un couvert de glace sur l'écoulement d'une rivière est assez bien connu (Ashton, 1986). Lorsque le couvert se bâtit ou se détruit, le débit peut diminuer ou augmenter mais lorsque le couvert est stable (bien formé et en très faible évolution), sa présence n'a pas d'impact sur le débit. Par contre, elle aura un impact sur les niveaux principalement parce que le couvert augmente la longueur du périmètre mouillé. 
Devant le fait qu'il y a deux fois plus de surface de frottement, pour véhiculer le débit imposé, le niveau de la rivière doit s'élever (ou, si possible, augmenter sa pente). Le montant de l'augmentation du niveau peut facilement se calculer à l'aide d'une équation telle que Manning ou Chézy. Si la résistance de la glace est le même que le fond et si la pente est invariante, en présence d'un couvert de glace, le rayon hydraulique est environ la moitié et alors pour un même débit, la profondeur doit augmenter de 32\%. Évidemment, sachant que le débit est le même, l'augmentation des niveaux se traduit par une réduction des vitesses moyennes, des forces de cisaillement et du transport de sédiments.

Ce résultat fondamental peut être modifié selon la valeur de Manning de la glace par rapport à la valeur de Manning du fond. Des études préliminaires d'Environnement Canada démontrent que la valeur de Manning $\mathrm{n}$ de la glace est normalement le tiers de celle du fond. Dans ce cas, la valeur composite est $70 \%$ de la valeur du fond et alors l'augmentation de la profondeur d'eau requise pour passer le même débit qu'en eau libre est de $8 \%$. Par contre, en présence d'embâcle, la rugosité de la glace peut être plusieurs fois celle du fond et les profondeurs d'eau dans ces situations peuvent être plusieurs fois la profondeur de celle en eau libre (Beltaos, 1995).

\subsection{Influence d'un couvert de glace sur l'écoulement dans un estuaire}

L'influence du couvert de glace dans un estuaire est beaucoup plus subtile. Il est le résultat d'une combinaison de plusieurs effets. Alors que dans les rivières, le débit et (normalement) la pente sont des valeurs imposées (et alors que la rivière est libre de s'ajuster son niveau), dans les estuaires, le niveau est imposé à l'embouchure et alors l'estuaire peut modifier les niveaux seulement en amont. Mais il peut aussi changer sa pente et son débit en tous temps et lieux. Considérons un élément à la fois...

Le niveau dans l'estuaire à la marée haute est inchangé en présence de couvert de glace car il n'a pas d'impact sur la résistance à l'écoulement à ce moment car le débit est nul. Le niveau à la marée basse est imposé à l'embouchure (qu'il ait de la glace ou non). Par contre, à l'amont, le niveau peu être supérieur car l'eau coule dans ces circonstances et le périmètre mouillé est deux fois plus grand.

Le volume du prisme en présence du couvert est seulement légèrement modifié en hiver. Premièrement, comme nous venons de voir, le niveau à la marée basse est un peu plus élevé en hiver. Deuxièmement, il y a un certain pourcentage de débit qui n'est pas évacué à la marée basse. Elle reste plutôt sur les les battures en forme solide (c'est-à-dire, au lieu de s'évacuer par le jusant, l'eau contenu dans le couvert de glace se repose sur le fond).

Qu'il y ait un couvert de glace ou non, le temps d'un cycle de marée ne change pas à l'embouchure mais la phase de la marée peut changer plus haut dans l'estuaire. Le débit $\approx$ (Volume du prisme) / (temps d'un demi cycle de marée) est alors influencé par la modification du prisme et de la déphasage des niveaux.

De plus, à cause de la résistance additionnelle induite par la présence du couvert de glace, il faut que la pente de l'estuaire augmente pour passer le débit. Cette augmentation de la pente se traduit par un retard dans la phase des niveaux dans l'estuaire.

Alors comme on peut constater, dans un estuaire, l'impact du couvert de glace est ressentit à la fois sur les niveaux (surtout à l'amont), sur le débit, sur la pente et sur la phase des marées.

Évidemment les vitesses moyennes (1-D) seront aussi modifiées car celles-ci dépendent directement des débits, des niveaux en plus que sur l'épaisseur du couvert (qui réduit l'aire d'écoulement pour une même charge d'eau).

Enfin, la distribution de la vitesse dans la colonne d'eau (2-D verticale) ne sera plus logarithmique car il y a, en présence du couvert, une distribution à deux parois. De plus la distribution transversale (2-D transversale) sera modifiée car le débit sur les zones en rives 
plus profondes est beaucoup influencé par l'épaisseur de la couverture de glace (qui est du même ordre de grandeur) que dans le chenal principal de l'estuaire.

Exactement comment tout va se traduire dans le concret dépend des caractéristiques de l'estuaire, des marées et du couvert de glace.

Le suivant est une présentation des simulations 1-D de l'estuaire Portneuf. Le modèle numérique utilisé ( ONE-D ») est celui développé au Massachusetts Institut of Technology et supporté par Environnement Canada. Il est dans le domaine public et il s'agit d'un modèle à éléments finis qui considère pleinement les équations non permanentes de Saint-Venant.

\section{Présentation du site}

\section{L'estuaire de la rivière Portneuf}

La côte Nord de la province de Québec est parcourue par de nombreuses rivières qui viennent se jeter dans l'estuaire du fleuve Saint-Laurent. La rivière Portneuf située à environ $260 \mathrm{~km}$ au nord-est de la ville de Québec fait partie de ces rivières. La rivière Portneuf dont le bassin versant s'étend sur $2100 \mathrm{~km}^{2}$, prend sa source dans le lac Itomamo et se jette

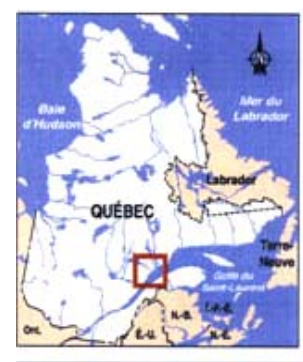
$185 \mathrm{~km}$ plus loin dans la partie maritime de l'estuaire du fleuve Saint-Laurent se plaçant ainsi en partie sous l'influence marémotrice du fleuve ayant environ $45 \mathrm{~km}$ de large. Les niveaux dans l'estuaire St. Laurent à cette hauteur sont entièrement prescrites par l'action des astres sauf en cas de tempêtes.Nous avons pris plusieurs relevés aux sites de la Portneuf identifiés sur la figure 1.

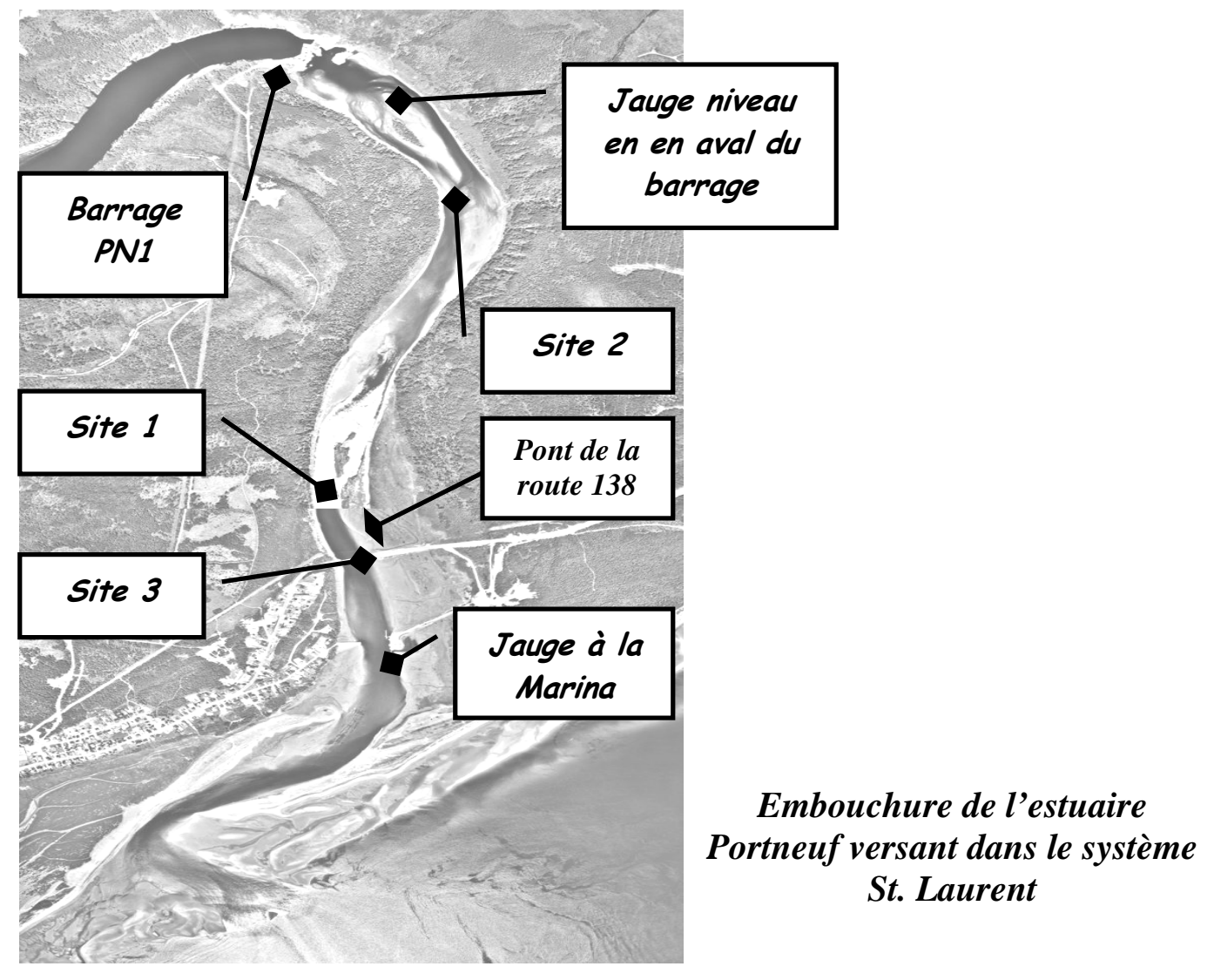

Figure 1 : Emplacement des 4 sites de l'estuaire Portneuf. (photo aérienne du 4 juillet 2000) 


\section{Résultats des simulations numériques}

\subsection{Calibration du modèle}

Afin de mettre en évidence l'influence du couvert de glace sur le régime hydraulique de l'écoulement, nous avons utilisé le modèle One-D en plaçant un couvert d'épaisseur variable de 0 à $50 \mathrm{~cm}$ (en fonction de l'avancement de l'hiver). Selon notre calibration, l'été, la valeur de Manning $n$ était 0,028 alors que l'hiver sa valeur composite (fond et glace ensemble) était de 0,025 . En général, la calibration était assez précise sauf proche de l'embouchure où le fond sableux et le couvert de glace consolidé sont toujours très variables.

\subsection{Les effets du couvert de glace sur les niveaux}

Nous avons donc effectué ces simulations avec et sans glace. A partir d'elles, nous avons donc pu faire ressortir certains impacts du couvert de glace sur l'hydrodynamique de l'estuaire (figure 2).

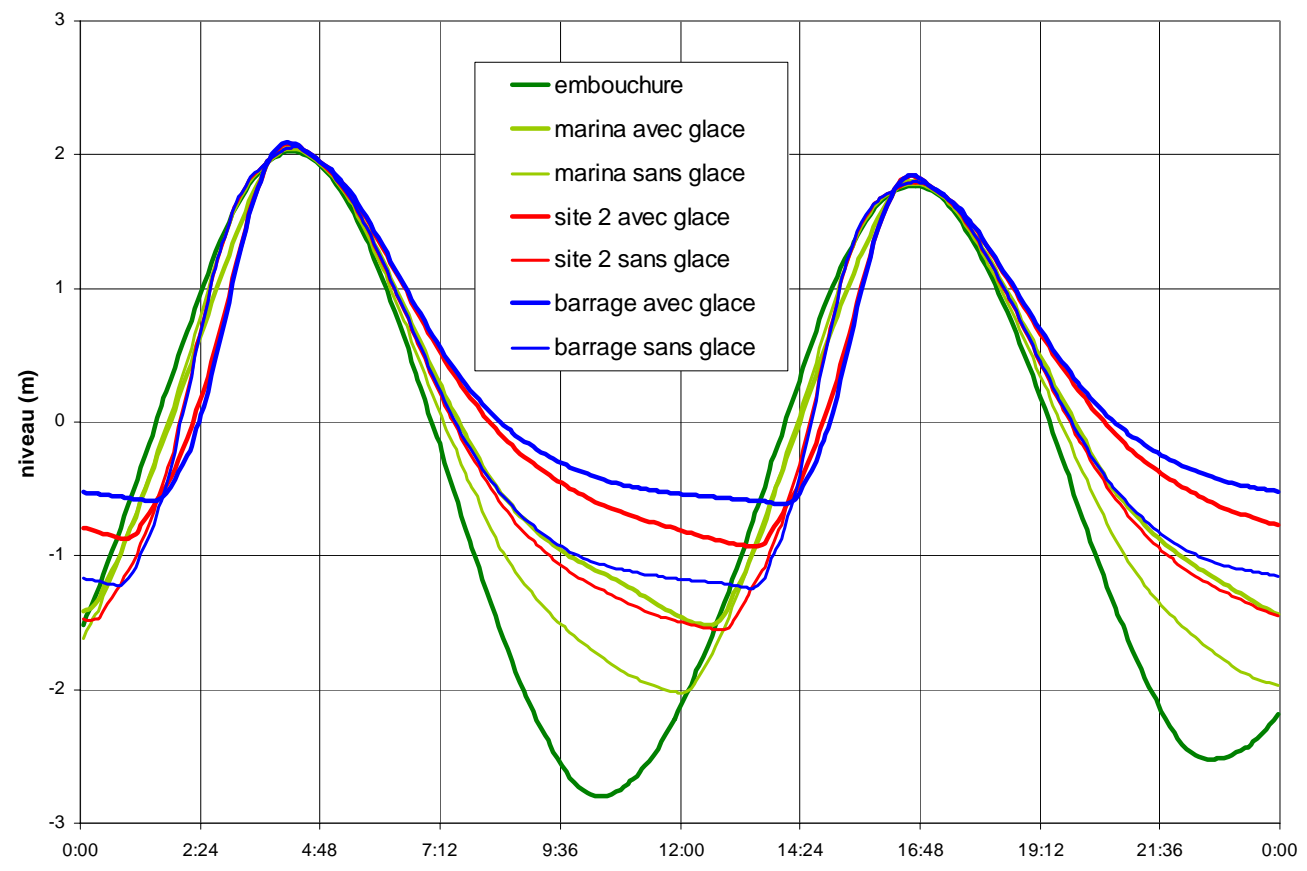

Figure 2 : Niveaux le 2 Mars 2002 selon le modèle One-D (couvert de glace de $40 \mathrm{~cm}$ à $50 \mathrm{~cm}$; débit turbiné de $13.5 \mathrm{~m}^{3} / \mathrm{s}$; marée vive).

La figure 2 présente les niveaux simulés en différents points de l'estuaire durant une journée de marée vive en présence et en absence du couvert de glace. Les niveaux à l'embouchure sont les mêmes dans les deux cas car ils ne dépendent aucunement des conditions de l'estuaire. Au contraire, ils représentent les niveaux de la marée qui se propage dans le St. Laurent. Autrement dit, ces niveaux sont imposés à l'estuaire et deviennent les conditions limites du modèle numérique ONE-D. (L'autre imposition de conditions limites est le débit turbiné au barrage).

A marée haute, à l'embouchure, les niveaux atteignent 2,0m et, au barrage, les niveaux maxima simulés atteignent $2,06 \mathrm{~m}$ qu'il y ait de la glace ou non. Les niveaux maximaux sont 
donc très similaires sur l'ensemble de l'estuaire et présence comme en absence de glace. A marée basse, c'est autre chose :

Tableau1. Niveaux minimaux en présence et absence d'un couvert de glace.

\begin{tabular}{|c|c|c|c|}
\hline \multicolumn{2}{|c|}{ Niveaux minimaux atteints à la marée basse en présence et absence d'un couvert de glace } \\
\hline Station & $\begin{array}{c}\text { Niveau minimal à la } \\
\text { marée basse atteint en } \\
\text { absence de glace } \\
\text { (conditions estivales) }\end{array}$ & $\begin{array}{c}\text { Niveau minimal à la } \\
\text { marée basse atteint } \\
\text { avec couvert de glace } \\
\text { (conditions } \\
\text { hivernales) }\end{array}$ & $\begin{array}{c}\text { Impact de la } \\
\text { présence du } \\
\text { couvert de glace } \\
\text { sur }\end{array}$ \\
\hline Embouchure : & $-2,8 \mathrm{~m}$ & $-2,8 \mathrm{~m}$ & l'hydrodynamique \\
\hline Marina : & $-2,0 \mathrm{~m}$ & $-1,5 \mathrm{~m}$ & $0,5 \mathrm{~m}$ \\
\hline Site no. 2: & $-1,5 \mathrm{~m}$ & $-0,9 \mathrm{~m}$ & $0,6 \mathrm{~m}$ \\
\hline Barrage : & $-1,2 \mathrm{~m}$ & $-0,6 \mathrm{~m}$ & $0,6 \mathrm{~m}$ \\
\hline
\end{tabular}

On note donc une augmentation des niveaux bas d'environ $50 \mathrm{~cm}$ en présence de glace sur l'ensemble de l'estuaire (Tableau 1). Cette augmentation de niveau se fait ressentir principalement entre l'embouchure et la marina mais elle est par ailleurs d'autant plus grande que l'on s'éloigne de l'embouchure (50 cm à la marina, $60 \mathrm{~cm}$ au barrage). Par ailleurs, on note un déphasage des niveaux bas (Tableau 2) :

Tableau 2. Arrivée de la marée basse en présence et absence de glace.

\begin{tabular}{|c|c|c|c|}
\hline \multicolumn{2}{|c|}{ L'heure de l'arrivée de la marée basse en présence et absence d'un couvert de glace } \\
\hline Station & $\begin{array}{c}\text { L'heure d'arrivée du } \\
\text { niveau bas en absence } \\
\text { de couvert de glace }\end{array}$ & $\begin{array}{c}\text { L'heure d'arrivée du } \\
\text { niveau bas en } \\
\text { présence de couvert } \\
\text { de glace }\end{array}$ & $\begin{array}{c}\text { Impact de la } \\
\text { présence du } \\
\text { couvert de glace }\end{array}$ \\
\hline Embouchure : & $10 \mathrm{~h} 23$ & $10 \mathrm{~h} 23$ & nul \\
\hline Marina : & $12 \mathrm{~h} 08$ & $12 \mathrm{~h} 48$ & 40 minutes \\
\hline Site no. 2: & $12 \mathrm{~h} 53$ & $13 \mathrm{~h} 38$ & 45 minutes \\
\hline Barrage : & $13 \mathrm{~h} 28$ & $14 \mathrm{~h} 18$ & 50 minutes \\
\hline
\end{tabular}

Le déphasage des niveaux bas est donc de 40 minutes à la marina et il augmente plus on s'approche du barrage.

La présence d'un couvert de glace va donc, dans un premier temps, entraîner une augmentation des niveaux d'eau à marée basse alors qu'il n'y a aucun changement notable à marée haute. Par ailleurs, les niveaux bas observés en présence de glace sont en retard par rapport à ceux observés en absence de glace. 
Une étude plus systématique sur l'ensemble de l'hiver nous a permis de dresser un profil approximatif de l'influence du couvert de glace en terme d'amortissement du marnage et de déphasage. Nous avons ainsi calculé, à partir des données simulées par le modèle One-D un amortissement du marnage en différents points de l'estuaire. Cet amortissement correspond au rapport de la différence de niveau bas avec et sans glace sur le marnage évalué au même point et au même instant (Tableau 3).

Tableau 3. Amortissement du marnage en présence du couvert de glace.

\begin{tabular}{|c|c|c|c|c|c|c|c|}
\hline \multirow[b]{2}{*}{ Période : } & \multirow[b]{2}{*}{$\begin{array}{l}\text { Épaisseur } \\
\text { du } \\
\text { couvert } \\
\text { de glace } \\
\text { à l'aval de } \\
\text { la marina }\end{array}$} & \multirow[b]{2}{*}{$\begin{array}{l}\text { Épaisseur } \\
\text { du } \\
\text { couvert } \\
\text { de glace } \\
\text { à l'amont } \\
\text { de la } \\
\text { marina }\end{array}$} & \multicolumn{5}{|c|}{ Amortissement du marnage $(\%)$} \\
\hline & & & $\begin{array}{l}\text { Marina } \\
(1750 \mathrm{~m} \\
\text { de } \\
\text { l'embou- } \\
\text { chure) }\end{array}$ & $\begin{array}{l}\text { Site } 1 \\
(2830 \mathrm{~m} \\
\text { de } \\
\text { l'embou- } \\
\text { chure) }\end{array}$ & $\begin{array}{l}\text { Site } 2 \\
(4030 \mathrm{~m} \\
\text { de } \\
\text { l'embou- } \\
\text { chure) }\end{array}$ & $\begin{array}{l}\begin{array}{l}\text { Avant le } \\
\text { barrage }\end{array} \\
(4895 \mathrm{~m} \\
\text { de } \\
\text { l'embou- } \\
\text { chure) }\end{array}$ & $\begin{array}{l}\mathrm{Au} \\
\text { barrage } \\
(5600 \mathrm{~m} \\
\text { de } \\
\text { l'embou- } \\
\text { chure })\end{array}$ \\
\hline $\begin{array}{l}1 \text { déc - } \\
16 \text { déc. }\end{array}$ & 0 & 3 & $0-1$ & $4-6$ & $6-9$ & $8-12$ & $8-12$ \\
\hline $\begin{array}{l}1 \text { jan. - } \\
20 \text { jan. }\end{array}$ & 0 & 15 & $0-1$ & $4-6$ & $7-10$ & $9-11$ & $9-12$ \\
\hline $\begin{array}{l}4 \text { fév. - } \\
28 \text { fév. }\end{array}$ & 50 & 50 & $4-13$ & $8-16$ & $10-22$ & $13-15$ & $18-29$ \\
\hline $\begin{array}{l}1 \text { mars - } \\
15 \text { mars }\end{array}$ & 40 & 50 & $4-14$ & $8-16$ & $15-25$ & $16-29$ & $17-37$ \\
\hline
\end{tabular}

\subsection{Les effets du couvert de glace sur les débits}

Sachant que la présence du couvert modifie les niveaux d'eau, les débits et courants vont aussi être modifiés.

La figure 3 représente l'influence de la glace sur le débit. Les valeurs négatives représentent ici les débits dans l'estuaire pour un écoulement allant du barrage vers l'embouchure lors du reflux (marée descendante). Les valeurs positives représentent alors les débits lors de la marée montante. Lors de cette simulation, nous avons imposé au modèle numérique, $40-50 \mathrm{~cm}$ de glace et un débit de $13.5 \mathrm{~m}^{3} / \mathrm{s}$ au barrage. Ce sont des conditions de marée vive. Nous notons que la présence du couvert a un impact sur l'amplitude du débit et sur le temps à laquelle les débits maximaux se rendent à une station (c'est-à-dire la phase). Dans le cas du 2 mars (vers 2h30) lors du flot, le débit à la marina est $393 \mathrm{~m}^{3} / \mathrm{s}$ en absence du couvert et seulement $347 \mathrm{~m}^{3} / \mathrm{s}$ en présence du couvert de glace. Ça représente un amortissement de 13\%. Le couvert cause aussi un retard de ce débit maximum de 45 minutes. 


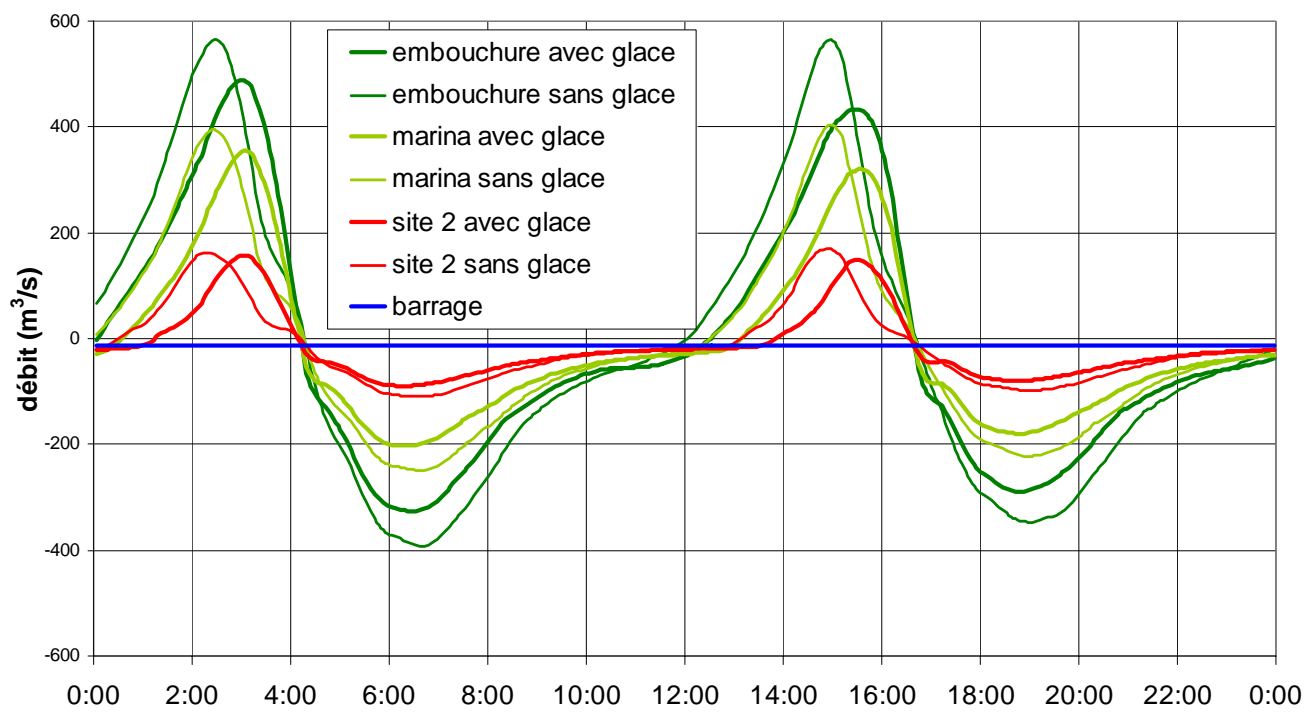

Figure 3 : Débits le 2 Mars 2002 selon le modèle One-D

Pour les débits lors du jusant, à la marina sans couvert de glace, il est $249 \mathrm{~m}^{3} / \mathrm{s}$ alors qu'en présence du couvert de glace, il n'est que $201 \mathrm{~m}^{3} / \mathrm{s}$. Ça représente une diminution de $19 \%$. Dans le cas des débits négatifs (lors du jusant), il semble que, selon le site, le couvert de glace peut avoir moindre d'impact sur la phase du signal du débit.

\subsection{Les effets du couvert de glace sur les vitesses}

Afin d'avoir une analyse complète de l'influence du couvert de glace sur l'hydrodynamique de l'estuaire, nous avons également étudié les vitesses dans l'estuaire en présence et en absence de glace grâce au modèle One-D.

En absence de glace, les vitesses varient de -141 à $81 \mathrm{~cm} / \mathrm{s}$ à 1 'embouchure, de -56 à $80 \mathrm{~cm} / \mathrm{s}$ à la marina et de -32 à $46 \mathrm{~cm} / \mathrm{s}$ au site 2 . En présence de glace, les vitesses varient de 142 à $61 \mathrm{~cm} / \mathrm{s}$ à 1 'embouchure, de -47 à $71 \mathrm{~cm} / \mathrm{s}$ à la marina et de -25 à $42 \mathrm{~cm} / \mathrm{s}$ au site 2 (Figure 4) : 


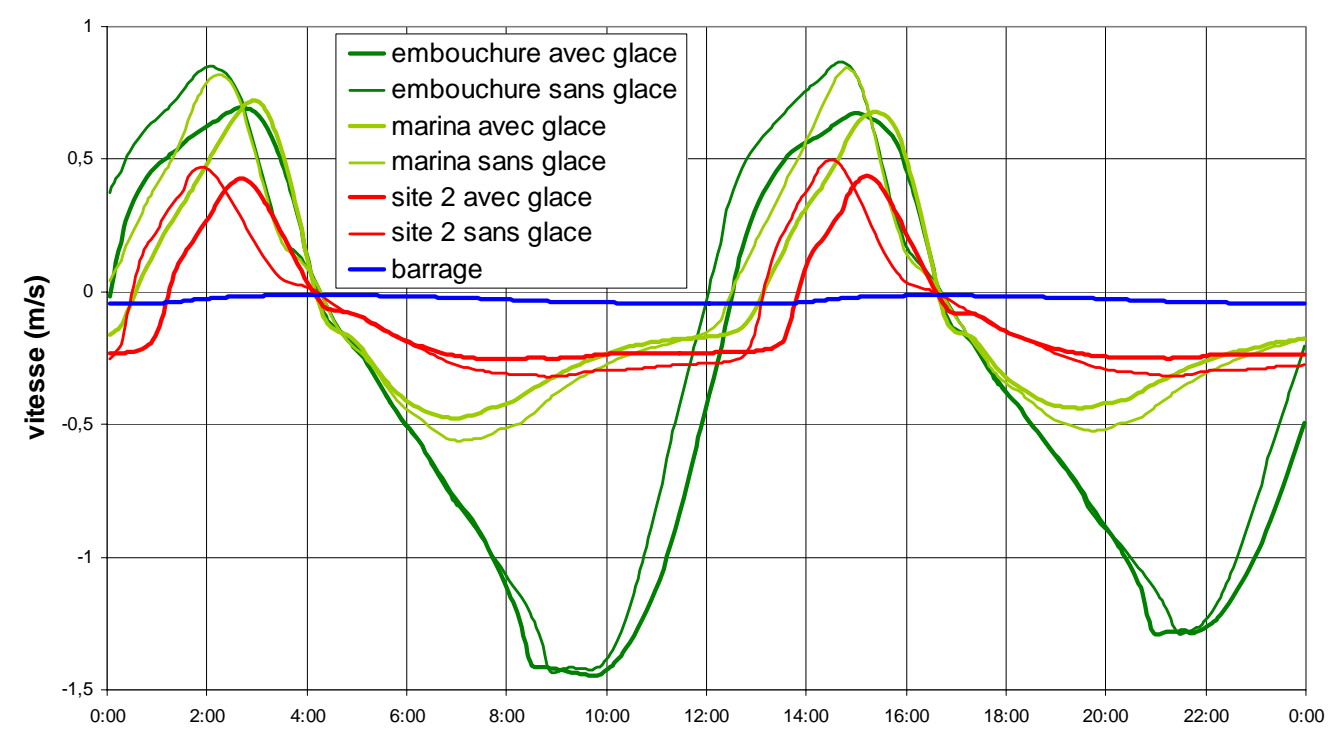

Figure 4 : Vitesses le 2 Mars 2002 selon le modèle One-D

(40 cm de glace sur le $1^{\text {er }}$ tronçon et $50 \mathrm{~cm}$ sur le reste, débit fluvial de $13.5 \mathrm{~m}^{3} / \mathrm{s}$, marée vive).

\section{Conclusions}

En présence de couvert de glace, le marnage est réduit jusqu'à $37 \%$ de sa valeur sans couvert dans la partie supérieure de l'estuaire. Plus le couvert est épais et étendu plus l'amortissement des niveaux est important.

En présence de couvert de glace, les débits sont diminués jusqu'à $27 \%$ de leur valeur en absence d'un couvert. En valeurs absolues, la glace semble avoir un impact plus important sur les débits lors du flux que lors du reflux, alors qu'en valeurs relatives, la glace aurait un impact plus important sur les débits lors du reflux que lors du flux.

Les vitesses sont elles aussi amorties par la présence d'un couvert de glace.

Le déphasage des niveaux bas atteint 86 minutes au barrage, celui des débits à l'embouchure atteint 90 minutes lors du flux et 45 minutes lors du reflux.

L'évaluation de l'impact du couvert de glace sur les processus sédimentaires est en dehors de notre étude. Par contre, sachant que le couvert de glace amortie le signal du niveau d'eau, du débit et des courants, nous présumons qu'il amortie aussi de façon significative le transport sédimentaire. Nous recommandons une extension de l'étude pour quantifier les processus sédimentaires hivernaux et pour les comparer avec ceux lors de la crue printanière et la période estivale.

Terminons en affirmant que les processus hivernaux dans l'estuaire de la Portneuf sont extrêmement fascinants. Chaque processus dépend des autres. Nous n'avons pas pu en parler mais rentre en ligne de compte aussi : la météo (température de l'air, humidité relative, vitesse du vent et quantité de neige); l'hydrologie (débit d'eau turbiné provenant du bassin); les cycles de marées basses, marées hautes, marées mortes et marées vives; les structures géomorphologiques; les caractéristiques de l'eau (salinité et température) de l'estuaire SaintLaurent (surtout en surface proche de la rive nord); et le transport littoral dans le SaintLaurent (fonction surtout des tempêtes). Le tout crée un système sédimentaire, hydrodynamique, thermodynamique et biologique vibrant et unique. 


\section{Remerciements}

Finalement, nous reconnaissons l'appui financier d'Hydro Québec, et l'appui technique et scientifique des ses employés, particulièrement Danielle Messier, Tung Thanh-Quach et Benoît Maisonneuve.

\section{Références}

1.Ashton, G.D. (1986). River and ice engineering. Water Resources Publications,Littleton, Colo.

2.Beltaos S. (1995). Ice jam processes. In River Ice Jams, Beltaos S (ed.). Water Resources Publications: Colorado; 71-104.

3.Morse, B., Burrell, B., St. Hilaire, A., Bergeron, N., Messier, D., Quach-Thanh,T. (1999). River ice processes in tidal rivers: Research needs. Proceedings of the $10^{\text {th }}$ Workshop on River Ice. June 8-11, 1999. Winnipeg. Manitoba. Canada. pp 388-399.

4.Morse, B., Messier, D., Stander, E. and Quach-Thanh,T. (2001). Le savoir écologique local de la dynamique des glaces dans l'estuaire Portneuf. 11th Workshop on River Ice. May 14-16, 2001. Ottawa, Ontario. Canada.

5.Morse, B., Messier, D., Stander, E. and Quach-Thanh,T. (2002). La dynamique hivernale de l'estuaire Portneuf. 36th Congress of the Canadian Meteorological and Oceanographic Society 2-5 May, 2002. Rimouski, Québec, Canada.

6.Morse, B., Stander, E., RINGO, B., Messier, D., Quach-Thanh, T. (2003). Ice formation and break-up in meso-tidal estuaries. 1ère Conférence spécialisée sur le génie Côtier, des estuaires et de l'offshore de la société canadienne de génie civil. 4-7 Juin 2003. Moncton, Nouveau Brunswick.

7.Savard, J-P. (1999). Étude comparative des estuaires nordiques, phase 4 : Standardisation de la méthode paramétrique. Hydro Québec, Unité Hydraulique et Environnement, mai 1999, 82p. 